\title{
Laser Triggered Injection of Electrons in a Laser Wakefield Accelerator with the Colliding Pulse Method
}

\author{
K. Nakamura*†, G. Fubiani* ${ }^{*}$, C. G. R. Geddes*8, P. Michel ${ }^{*}$, \\ J. van Tilborg*II C. Tóth*, E. Esarey*, C. B. Schroeder* and W. P. Leemans* \\ ${ }^{*}$ Lawrence Berkeley National Laboratory, University of California, Berkeley, CA 94720 \\ ${ }^{\dagger}$ University of Tokyo, Japan \\ \#niversity of Paris XI, Orsay, France \\ ${ }^{\S}$ Department of Physics, University of California, Berkeley, $C A$ \\ "Technische Universiteit Eindhoven, the Netherlands
}

\begin{abstract}
An injection scheme for a laser wakefield accelerator that employs a counterpropagating laser (colliding with the drive laser pulse, used to generate a plasma wake) is discussed. The threshold laser intensity for electron injection into the wakefield was analyzed using a heuristic model based on phase-space island overlap. Analysis shows that the injection can be performed using modest counterpropagating laser intensity $a_{1} \leq 0.5$ for a drive laser intensity of $a_{0} \simeq 1$.0. Preliminary experiments were preformed using a drive beam and colliding beam. Charge enhancement by the colliding pulse was observed. Increasing the signal-to-noise ratio by means of a preformed plasma channel is discussed.
\end{abstract}

\section{INTRODUCTION}

Laser-driven plasma-based accelerators operating in the self-modulated regime (for a review, see Ref. [1]) have succeeded in producing electron beams containing up to multi$\mathrm{nCs}$ of charge with energies in excess of 10's of MeV [2]. Due to the uncontrolled electron injection into the plasma wave, characteristic of the self-modulated regime, the energy distribution contains $100 \%$ energy spread. Generating electron beams with narrow energy spread (few \%) is required for many applications. As has recently been shown [3], through careful control of the acceleration length, laser and plasma parameters, electron beams with \%-level energy spread can be produced. Control of the trapping process is needed to generate monoenergetic electron beams in a reproducible manner. Here we discuss progress on the use of colliding pulse injection (CPI) for stabilizing the production of monoenergetic electron beams. Electron injection into the accelerating field of the plasma wave is problematic using conventional RF technology, since the characteristic scale length of the wake field in a plasma-based accelerator is the plasma wave length $\lambda_{p}$ (typically $\leq 100 \mu \mathrm{m}$ ), i.e., much shorter than conventional RF accelerators. In addition, to perform with good pulse-to-pulse energy stability, the femtosecond synchronization between the injection process and the phase of accelerating field is required, which is beyond the performance of current conventional accelerator technology.

All-optical schemes to perform such a highly precise injection have been proposed 
$[4,5]$ which rely on laser trigged injection of plasma electrons into a plasma wakefield. The colliding pulse injection (CPI) scheme [5] relies on the ponderomotive force associated with the beating of two lasers to inject electrons into the plasma wave. This CPI method requires lower laser intensity, compared to the LILAC scheme [4] which uses the ponderomotive force associated with a laser envelope.

In this paper we consider a CPI configuration in which the electron injection results from the beat wave generated by a counterpropagating injection pulse and the trailing part of the drive laser pulse $[6,7]$. In the following, analytical models, simulations, and preliminary experimental results of two-pulse CPI concept are presented and discussed.

\section{PHASE SPACE ANALYSIS OF TWO-PULSE CPI}

The injection threshold for two-pulse CPI scheme is analyzed by considering the motion of electron in phase space. Hamiltonians describing the electron behavior in the wakefield and the beat wave are derived individually, and particle trapping from the background plasma by the laser beat wave is examined using a phase-space island overlap criterion for the two waves [8].

In the absence of the beat wave (or counterpropagating, colliding laser pulse), the electron motion in a one-dimensional plasma wake is described by the Hamiltonian [6]

$$
H\left(u_{z}, \psi\right)=\sqrt{\gamma_{\perp}^{2}(\psi)+u_{z}^{2}}-\beta_{\phi} u_{z}-\phi(\psi)
$$

where $\boldsymbol{u}=\boldsymbol{p} / m_{e} c$ is the normalized electron momentum, $\psi=k_{p}\left(z-v_{\phi} t\right)$ is phase of the plasma wave, $k_{p}=\omega_{p} / c$ is the plasma wavenumber, $v_{\phi}=c \beta_{\phi}$ is the phase velocity of the plasma wave, which is near the group velocity of laser pulse $v_{g} \simeq c \beta_{\phi}$, $\gamma_{\perp}^{2}=\gamma^{2} / \gamma_{z}^{2}=1+a^{2}, a$ is the normalized vector potential of laser pulse $\boldsymbol{a}=e \mathbf{A} / m c^{2}$, and $\phi(\psi)$ is the laser-driven plasma wave potential,

$$
\phi(\zeta)=k_{p} \int_{0}^{\zeta} d \zeta^{\prime} \sin k_{p}\left(\zeta-\zeta^{\prime}\right) a^{2}(\zeta) / 4
$$

where $\zeta=z-v_{g} t \simeq z-v_{\phi} t$. The normalized axial momentum of the electron in an orbit ( $H=$ constant $)$ of the plasma wake is

$$
u_{z}(\psi)=\beta_{\phi} \gamma_{\phi}^{2}(H+\phi) \pm \gamma_{\phi} \sqrt{\gamma_{\phi}^{2}(H+\phi)^{2}-\gamma_{\perp}^{2}}
$$

where $\gamma_{\phi}^{2}=1 /\left(1-\beta_{\phi}^{2}\right)$. The background electron fluid motion in the plasma wave is defined by $H=1$.

Neglecting the wake field generated by the main drive pulse, the motion of the electron under the beat wave alone is described with the beat wave Hamiltonian [5]

$$
H_{b}\left(u_{z}, \psi_{b}\right)=\sqrt{\gamma_{\perp b}^{2}\left(\psi_{b}\right)+u_{z}^{2}}-\beta_{b} u_{z},
$$

where $\psi_{b}=\Delta k\left(z-\beta_{b} c t\right)$ is the beat wave phase, $\Delta k=k_{0}-k_{1} \approx 2 k_{0}$, with the notation 0 and 1 for drive beam and colliding beam, respectively, $\gamma_{\perp b}^{2}\left(\psi_{b}\right) \simeq 1+a_{0}^{2}+2 a_{0} a_{1} \cos \psi_{b}$ 

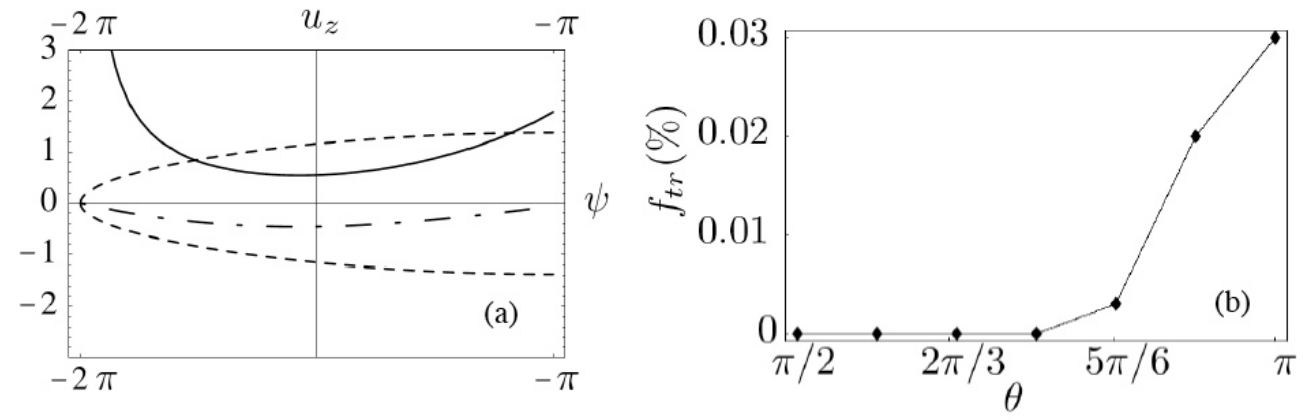

FIGURE 1. (a) Phase space $\left(\psi, u_{z}\right)$ showing trapped and focused separatrix (solid line), cold fluid orbit (dashed line), and maximum of the beat wave separatrix (dot-dashed line) for $a_{0}=0.95, a_{1}=0.5, \beta_{b}=0$, and $L_{0}=\lambda_{p}$. (b) Trapping fraction $f_{t r}$ as a function of angle for two laser beams with equal polarization at $\omega_{p} t=50$ with $a_{0}=1.0, \omega_{0} / \omega_{p}=50, L_{0}=9 \lambda_{p} / 8, a_{1}=0.5, \omega_{1} / \omega_{p}=50$, and $L_{1}=\lambda_{p} / 2$.

(where $a_{1} \ll 1$ is assumed), and $c \beta_{b}=\Delta \omega / \Delta k \ll c$ is the beat wave phase velocity $\left(\Delta \omega=\omega_{0}-\omega_{1}\right)$. The normalized axial momentum of an electron under the beat wave potential is

$$
u_{z b}\left(\psi_{b}\right)=\beta_{b} \gamma_{b}^{2} H_{b} \pm \gamma_{b} \sqrt{\gamma_{b}^{2} H_{b}^{2}-\gamma_{\perp b}^{2}} .
$$

By using of a phase-space separatrix overlap condition [8], the threshold laser intensity for electron injection into the wakefield can be estimated. Phase space $\left(\psi, u_{z}\right)$, with the trapped and focused separatrix, the untrapped electron orbit, and the maximum of the beat wave separatrix, is shown in Fig. 1(a). If the beat wave separatrix overlaps with the trapped wake orbit and the background electron orbit, an electron can be accelerated (and dephased) such that the background electrons become trapped. Overlap in phase space requires

$$
\begin{aligned}
& u_{b(\max )} \geq u_{z(\text { trap })}, \\
& u_{b(\min )} \leq u_{z(H=1)} .
\end{aligned}
$$

For the case of the counterpropagating colliding laser beam, trapping thresholds can be determined by solving Eqs. (6) and (7) for the normalized vector potentials of laser beams $a_{0}$ and $a_{1}$. Solving Eqs. (6) and (7) for several drive laser beam lengths with $\beta_{b}=0.05$ and $\psi=-3 \pi / 2$ (near the back of the drive pulse), the lowest threshold is obtained in the resonant case $\left(L=\lambda_{p}\right)$ where the largest wake field amplitude is produced. Also, the injection can be performed using modest laser intensity $a_{1} \leq 0.5$ for drive laser intensities $a_{0} \simeq 1.0$ [6]. Estimates based on the above Hamiltonian analysis have been shown to qualitatively agree with particle-in-cell simulations [7]. As shown in following section, experiments were performed having an angle of $5 \pi / 6$ between the two colliding laser beams. Therefore, the effects of interaction angle were examined. Figure 1(b) shows the trapping fraction (the fraction of the initial electrons that remain on trapped and focused orbits) versus interaction angle at $\omega_{p} t=50$ with $a_{0}^{2}=0.5, \omega_{0} / \omega_{p}=50, L_{0}=9 \lambda_{p} / 8, a_{0}^{2}=0.125, \omega_{1} / \omega_{p}=50$, and $L_{1}=\lambda_{p} / 2$. One can see that the trapping fraction decreases to zero as the angle $\theta$ decreases from $\pi$ to $\pi / 2$, 
and $15 \%$ of the electrons are trapped for the case of $5 \pi / 6$ interaction angle compared to the case of head-on collision $(\pi)$.

\section{EXPERIMENTAL SETUP AND MEASUREMENTS}

The experiments described in this paper were based on the $10 \mathrm{~Hz} \mathrm{Ti:} \mathrm{Al}_{2} \mathrm{O}_{3} \mathrm{CPA}$ laser system at the Lawrence Berkeley National Laboratory [9]. Low energy laser pulses from the oscillator (of wavelength $\lambda \simeq 800 \mathrm{~nm}$ ) were first temporally stretched, and split into two pulses. One was amplified up to $1 \mathrm{~J} /$ pulse level for driving a plasma wave, and the other was amplified to $0.3 \mathrm{~J} /$ pulse (colliding pulse). The drive and colliding beams were focused to a spot size $6 \mu \mathrm{m}$ with the $30 \mathrm{~cm}$ focal length off-axis parabolas (OAP0 and OAP1, respectively). The colliding beam intersected the drive beam from the downstream direction at a 30 degree angle. The schematic of the experiment is shown in Fig. 2.

The CPI experiments were performed with the drive and colliding laser beams focused onto a $2 \mathrm{~mm}$ long supersonic helium gas jet backed with up to 70 bar. The laser beam intensities were $a_{0}=1.3$ and $a_{1}=0.78$, to meet the analytical prediction of electron injection into the laser wakefield. The electron beam parameters were measured using an integrating current transformer (ICT) and a magnetic dipole spectrometer. As a rough measure of electron energy, the gamma ray yield, produced through Bremsstrahlung of the accelerated electrons when stopped in a high $\mathrm{Z}$ material, was also recorded using an ionization detector. The density profile of the plasma was measured using side-on interferometry of the folded-wave type, using a $400 \mathrm{~nm}$ wavelength, $50 \mathrm{fs}$ duration laser pulse. The interferometer was also used for temporal and spatial alignment of the two laser beams. Horizontal alignment was performed using plasma recombination radiation from the top view of the interaction point. Measured electron charge is shown in Fig. 3(a)

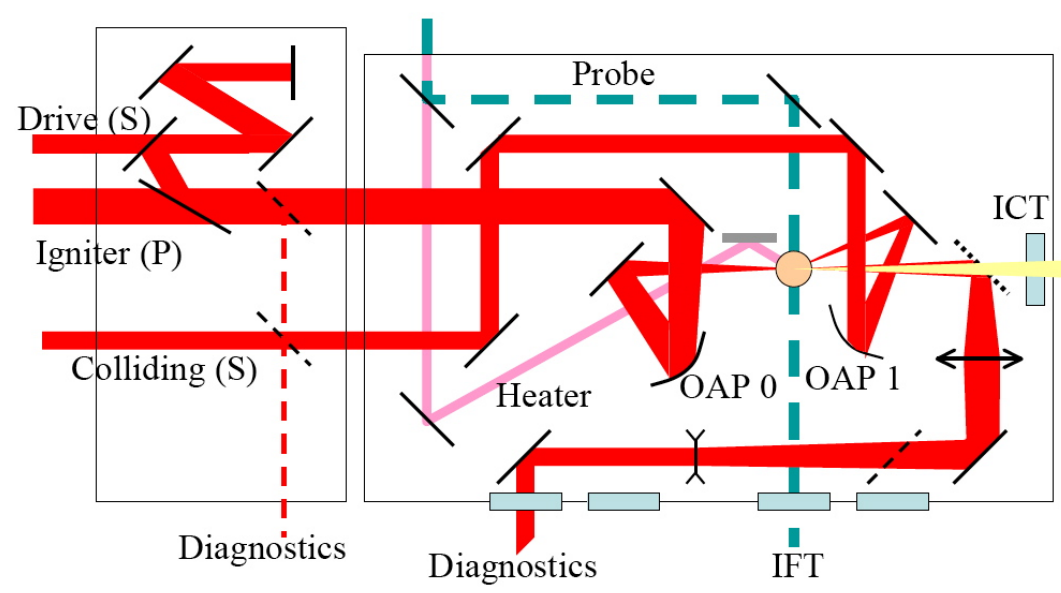

FIGURE 2. Schematic of the experimental setup. The high power laser beams are focused using an off-axis parabolic mirrors (OAP) onto a high pressure pulsed gas jet operating with about 70 bar helium backing pressure, respectively. An integrating current transformer (ICT) is used to measure the charge per bunch of the electron beam and plasma densities are measured with the interferometer (IFT). 
for the cases with and without the colliding laser pulse. One can see the enhancement of the acquired charge by the colliding laser beam and the dark current produced from the drive pulse without the colliding pulse. To verify CPI as the source of the enhancement, the time delay dependence of the current was measured and shown in Fig. 3(b). We expect the number of trapped electrons $N_{t r}$ to show a dependence on the delay time $\tau_{d}$, $N_{t r} \propto a_{0} \cdot a_{1}\left(\tau_{d}\right)$, since two-pulse CPI relies on the beat produced by the driving and the colliding laser beams. Therefore, one expects that the $N_{t r}$ has a maximum as a function of $\tau_{d}$ whose width is of the order of the length of the colliding beam. The result is shown in Fig. 3(b) and does not show sensitive dependence (on the ps-scale) of the electron yield nor gamma ray yield on the time delay between the two laser beams. This may be a result of the rather rough time scan as well as the low signal-to-noise $(\mathrm{S} / \mathrm{N})$ ratio between electrons generated by wavebreaking and CPI.

In order to increase the $\mathrm{S} / \mathrm{N}$ ratio, we are designing a future $\mathrm{CPI}$ experiment system that will use a preformed plasma channel generated by the ignitor-heater method [10] in conjunction with a slit nozzle $(200 \mu \mathrm{m} \times 4 \mathrm{~mm})$ gas jet. The CPI scheme is based on the standard laser wakefield regime with laser trigged injection [5]. Extended interaction length requires a preformed plasma channel since pulses in the standard regime will not self-guide. Using a preformed plasma channel, the resonant drive pulse is expected to generate a wakefield inside the plasma channel without trapping significant amounts of background electrons. Using CPI in a preformed plasma channel, we expect to produce narrow energy spread electron bunches. Fluctuations of the mean electron bunch momentum is also expected due to the pointing instability of lasers. A magnetic spectrometer, with large momentum acceptance $(80 \mathrm{MeV}$ to $1 \mathrm{GeV}$ with maximum excitation current), is also under development to characterize the electron bunches in a single-shot.

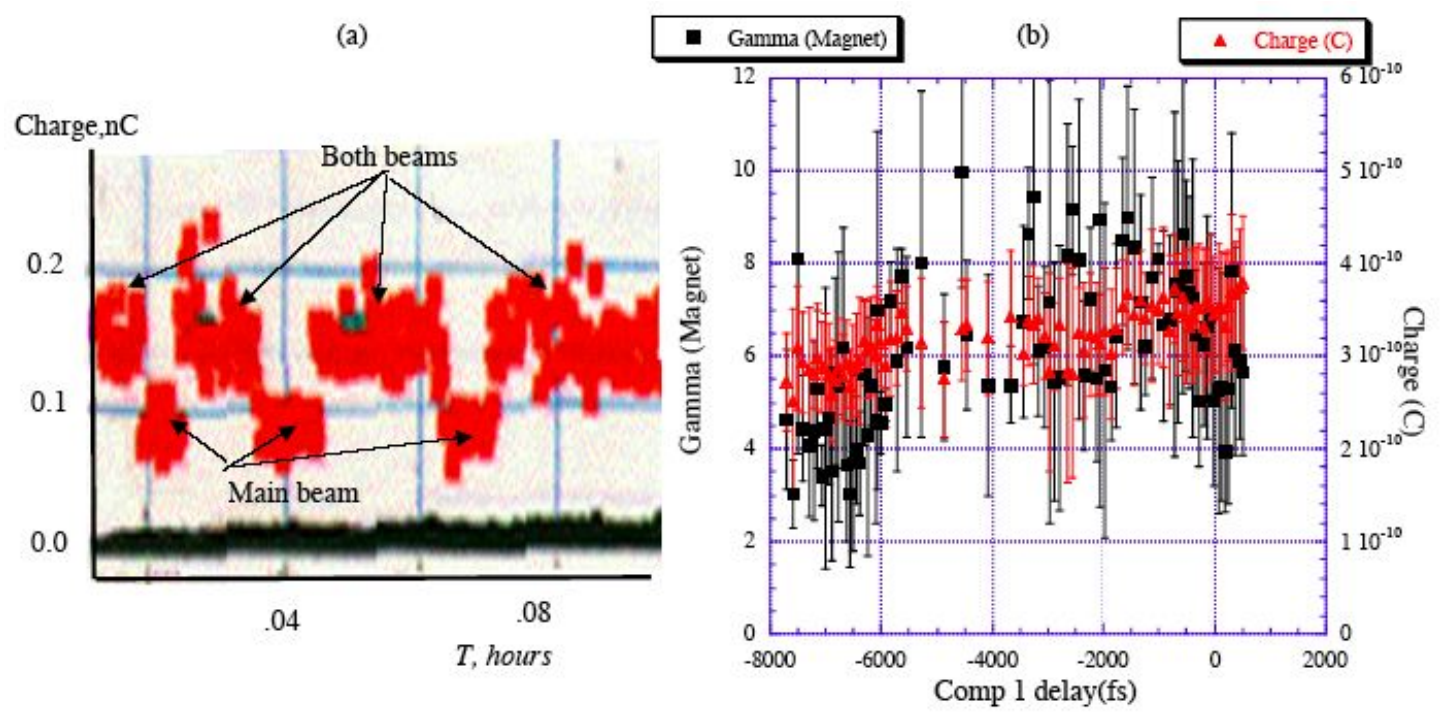

FIGURE 3. (a) Enhanced electron yield by colliding laser beam. Measured charges are shown with and without the colliding beam. (b) Charge and gamma ray yield as a function of time delay of colliding laser beam. 


\section{SUMMARY}

We have discussed the two-pulse colliding pulse injection method and preliminary experiments. The analysis indicates that injection can be performed at modest laser intensity $a_{1} \leq 0.5$ for a drive laser intensity of $a_{0} \simeq 1.0$. In the experiments using a 30 degree interaction geometry, the enhancement of accelerated electron generation using a colliding laser beam was observed. Future CPI experiments, that will utilize a preformed plasma channel and a large momentum acceptance magnetic spectrometer to obtain a high $\mathrm{S} / \mathrm{N}$ ratio, were also discussed.

\section{ACKNOWLEDGMENTS}

This work has been supported by U.S. Department of Energy under contract No DEAC-03-76SF0098. C. G. R. Geddes acknowledges support from the Hertz Foundation.

\section{REFERENCES}

1. E. Esarey, et. al., IEEE Trans. Plasma Sci. 24, 252 (1996).

2. W. P. Leemans, et. al, Phys. Rev. Lett. 89, 174802 (2002); V. Malka, et. al, Science 298, 1596 (2002); D. Umstadter, et. al, Science 273, 472 (1996); T. Hosokai, et. al, Phys. Rev. E 67, 036407 (2003).

3. C.G.R. Geddes et. al, Nature, to be published; these proceedings.

4. D. Umstadter, et. al, Phys. Rev. Lett. 76, 2073 (1996); R. G. Hemker, et. al, Phys. Rev. E 57, 5920 (1998).

5. E. Esarey, et. al, Phys. Rev. Lett. 79, 2682 (1997); C. B. Schroeder, et. al, Phys. Rev. E 59, 6037 (1999); E. Esarey, et. al, Phys. Plasmas 6, 2262 (1999).

6. G. Fubiani, et. al, Phys. Rev. E 79, 016402 (2004).

7. H. Kotaki, et. al, Phys. Plasmas 11, 3296 (2004).

8. A. J. Lichtenberg and M. A. Liberman, Regular and Stochastic Motion, Springer-Verlag, New York (1983).

9. W. P. Leemans, et. al, Phys. Plasmas 11, 2899 (2004).

10. P. Volfbeyn, et. al, Phys. Plasmas 6, 2269 (1999). 